\title{
REVIEW
}

\section{ESTADO DEL CONOCIMIENTO SOBRE EL CICLO REPRODUCTIVO Y CAPTACIÓN DE POSTLARVAS DE MEJILLÓN DEL GÉNERO Mytilus EN LAS COSTAS DE LA ARGENTINA}

\author{
TOMÁs E. ISOLA ${ }^{1,2}$ \\ ${ }^{1}$ Instituto de Desarrollo Costero, Universidad Nacional de la Patagonia San Juan Bosco, \\ Ciudad Universitaria km 4, Comodoro Rivadavia, Argentina \\ ${ }^{2}$ Consejo Nacional de Investigaciones Científicas y Técnicas (CONICET), Argentina \\ correo electrónico: isolatomas@yahoo.com.ar
}

\begin{abstract}
RESUMEN. El trabajo consiste en una revisión de las investigaciones realizadas en la Argentina sobre el ciclo reproductivo del mejillón Mytilus spp. y la captación de sus postlarvas en colectores artificiales en ambiente natural. Su principal objetivo es unificar, sistematizar y discutir los resultados obtenidos durante las últimas cuatro décadas de estudios sobre el tema. El enfoque abarca la perspectiva biológica y biotecnológica vinculada al cultivo de esta especie en el país.
\end{abstract}

Palabras clave: Colectores, Mytilus, captación, producción, cultivo.

\section{STATE OF KNOWLEDGE ABOUT THE REPRODUCTIVE CYCLE AND SETTLEMENT OF MUSSEL POSTLARVAE OF THE Mytilus GENUS OF THE COASTS OF ARGENTINA}

\begin{abstract}
The work comprises a review of the research carried out in Argentina on the reproductive cycle of the mussel Mytilus spp. and the settlement of its postlarvae in artificial collectors in natural environment. Its main objective is to unify, systematize and discuss the results obtained during the last four decades of studies on the subject. The approach covers the biological and biotechnological perspective linked to the cultivation of this species in the country.
\end{abstract}

Key words: Collectors, Mytilus, settlement, production, culture.

Esta revisión se basa en los trabajos de investigación realizados a lo largo de las costas del Mar Argentino, en relación al ciclo reproductivo del mejillón y el asentamiento de postlarvas en colectores artificiales destinados a la acuicultura. Su objeto fundamental es compilar la labor que han realizado numerosos investigadores a lo largo de más de cuatro décadas. Se incluyen además los resultados de tesis doctorales y otros formatos publicados en revistas científicas o informes téc- nicos. Se pretende establecer una relación entre los resultados que se obtuvieron en la temática referida, a los efectos de generar un documento que funcione como soporte para futuras investigaciones relativas al ciclo de vida del mejillón y su cultivo en la Argentina.

El mejillón que habita en las costas de la Argentina e Islas Malvinas pertenece al Género Mytilus. Aún existe controversia respecto de la identidad genética de las poblaciones presentes en la Argen- 
tina. Estudios recientes indican la presencia de individuos de Mytilus galloprovincialis e hibridación con el complejo M. edulis en el Golfo Nuevo (Zbawicka et al. 2018). Más allá de esta situación, tanto M. platensis (d'Orbigny 1842) como M. chilensis (Hupé 1856) fueron considerados como especies o subspecies de M. edulis (Linnaeus, 1758) (Castellanos 1957; Dell 1964; Seed 1990). Borsa et al. (2012) asume que M. edulis del hemisferio sur y $M$. chilensis deben ser asignados con un rango de subespecie y ambas especies deben ser consideradas bajo un mismo taxón. Por otra parte, Astoga et al. (2015) no encontró diferencias genéticas entre $M$. chilensis y $M$. platensis, es por esto que ambas son consideradas como subespecies y deberían ser agrupadas bajo M. edulis platensis de acuerdo con las prioridades que fija la nomenclatura zoológica. Por su parte Zbawicka et al. (2018) realizaron estudios genéticos sobre poblaciones de distintos puntos del litoral argentino y chileno en relación a colecciones de referencia y encontraron que $M$. edulis platensis e $M$. edulis chilensis serían especies distintas, estos resultados fueron obtenidos utilizando marcadores moleculares diferentes que en los estudios previamente mencionados aquí. Estas controversias hacen que el estatus taxonómico se encuentre en revisión y aún no esté aclarado. Por otra parte, los trabajos de investigación presentados aquí fueron realizados en diferentes temporalidades y cada uno de ellos está basado en la descripción y claves de identificación disponibles en ese momento, por lo que correspondería agruparlos en torno al Género Mytilus para evitar confusiones.

El cultivo de mejillón es un recurso económico en muchos países del mundo, donde distintas especies son cultivadas en función de su distribución geográfica natural, constituyendo una fuente importante de ingresos (Wijsman et al. 2019). La producción mundial de mejillón de cultivo es de dos millones de toneladas y genera ventas por cuatro mil millones de dólares americanos. El mayor productor es China (927.609 t), seguido por Chile (341.427 t), España (241.785 t) y Nueva Zelanda
(99.716 t) (FAO 2019). En el caso de la Argentina, el cultivo de mejillón $M$. edulis platensis es una actividad reconocida como de elevada potencialidad productiva y ha sido contemplada como eje estratégico de desarrollo científico nacional (Argentina Innovadora 2020); sin embargo, los volúmenes de cultivo de bivalvos en su conjunto nunca superaron las $100 \mathrm{t}_{\mathrm{año}}{ }^{-1}$, decreciendo hacia 2016 en una producción anual de 11,2 t (Panné Huidobro 2016). A partir de la década de los setenta se han desarrollo distintos emprendimientos de cultivo en la Argentina desde el sector público, privado y público-privado. Estas experiencias se han discontinuado por diversas razones, dentro de las cuales pueden mencionarse la inexistencia de un marco legal que reglamente la actividad, dificultades en las cadenas de comercialización, problemáticas ambientales (como por ejemplo, fenómenos prolongados de marea roja), la falta de clasificación de zonas aptas para cultivo, inexistencia de centros de expedición, interacción con actividades turísticas y servicios ambientales y robo de las producción, entre otras razones (RMCP 2013). No es la intención de este trabajo ahondar en la problemática de desarrollo específica del sector, sino resumir las investigaciones realizadas en torno a la reproducción del mejillón y su asentamiento en colectores dispuestos para su cultivo, haciendo hincapié en aspectos biológicos y biotecnológicos.

\section{CICLO REPRODUCTIVO Y CAPTACIÓN DE POSTLARVAS EN AMBIENTES NATURALES}

El asentamiento de postlarvas de mejillón en colectores artificiales en el ambiente natural constituye una significativa fuente de provisión de juveniles para países donde se desarrolla la acuicultura (Kamermans y Capelle 2019). El ciclo reproductivo de Mytilus spp. y su correlación con los períodos de captación varía a lo largo de la costa argentina (Figura 1). En la costa 


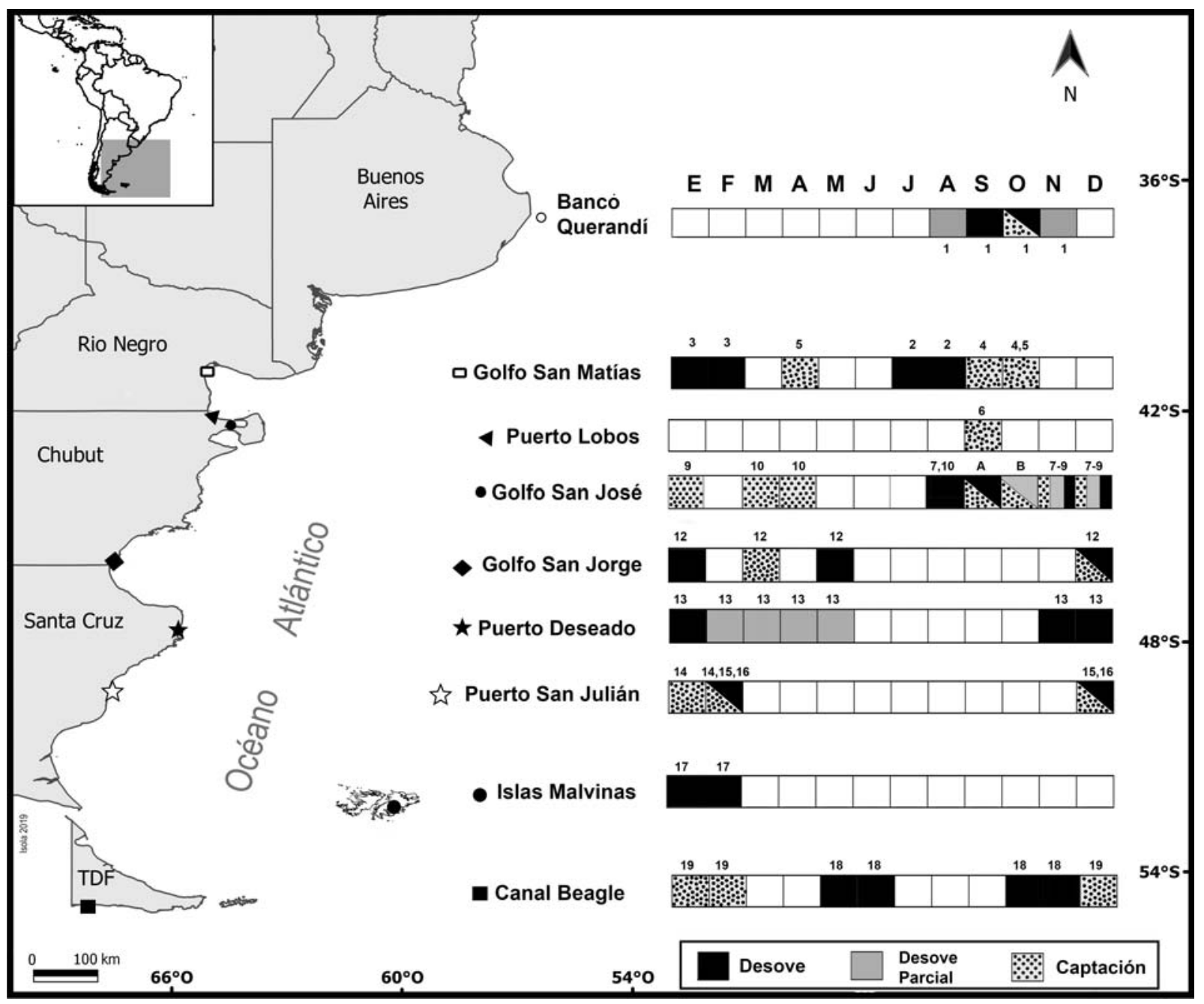

Figura 1. Ubicación geográfica de los sitios de estudio y resultados asociados por mes y temática. Se especifica en un calendario lo ubicación de los eventos de desove total, parcial y captación junto con el listado de autores por localidad estudiada. En algunos meses se encuentran más de un estudio en la tres diferentes temáticas, por lo tanto, se subdividieron los compartimentos en tantas partes como temáticas fueron estudiadas para ese mes y sitio. 1: Penchazadeh (1971, 1974), 2: Calvo et al. (1998), 3: Oehrens Kissner y Kroeck (2005), 4: Pascual y Zampatti (1998), 5: Elvira et al. (2000), 6: Agulleiro (2019), 7: Trancart (1978), 8: Bala (1989), 9: Ruzzante y Toyos de Guerrero (1984), 10: Lizarralde (1997), 11: Manriquez (2019), 12: Isola (2017), Isola et al. (2018), 13: Vinuesa (1978), 14: Martin el al. (2015), 15: Sar et al. (2018), 16: Zaixso et al. (2008), 17: Gray et al. (1997), 18: Tortorelli (1987), 19: Zampatti (2002), A: autores 6, 7, 8, 10 y 11, B: autores $8,10,11$.

Figure 1. Geographic location of study sites and results associated per month and subject. The location of the total, partial spawning and catchment events and the list of authors per location studied is specified in a calendar. Months in which more than one study is found in the three different themes, the compartments were subdivided into as many parts as the themes were studied for that month and site. 1: Penchazadeh (1971, 1974), 2: Calvo et al. (1998), 3: Oehrens Kissner y Kroeck (2005), 4: Pascual y Zampatti (1998), 5: Elvira et al. (2000), 6: Agulleiro (2019), 7: Trancart (1978), 8: Bala (1989), 9: Ruzzante y Toyos de Guerrero (1984), 10: Lizarralde (1997), 11: Manriquez (2019), 12: Isola (2017), Isola et al. (2018), 13: Vinuesa (1978), 14: Martin el al. (2015), 15: Sar et al. (2018), 16: Zaixso et al. (2008), 17: Gray et al. (1997), 18: Tortorelli (1987), 19: Zampatti (2002). A: authors 6, 7, 8, 10 and 11, B: Authors 8, $10,11$. 
bonaerense, el ciclo reproductivo fue estudiado por Penchaszadeh $(1971,1974)$, en base al análisis histológico y citometría de oocitos de individuos adultos recolectados en fondos circalitorales de 36-54 $\mathrm{m}$ de profundidad. Para su categorización, utilizó la escala propuesta por Chipperfield (1953), redefinida más tarde por Lubet (1959), pero simplificada. El autor halló maduraciones sincrónicas en la población con picos de desove en los meses de septiembre y octubre, aunque en algunos individuos ocurren en agosto y noviembre. Este período de desove se registró cuando la temperatura del agua de mar comenzó su ascenso a partir de $\operatorname{los} 10^{\circ} \mathrm{C}$. Luego se inicia una fase de reabsorción de elementos gonadales y reposo sexual que se extiende desde diciembre hasta abril o mayo. Los pulsos de desove fueron correlacionados con asentamientos masivos de juveniles en los bancos submareales a finales de octubre. El análisis de las tallas obtenidas en un muestreo de bancos infralitorales en la zona del faro Querandí indicó la presencia de juveniles cuyo asentamiento ocurrió en primavera (Lasta et al. 1986). Análisis realizados sobre el ciclo anual de los principales componentes bioquímicos indican una pérdida importante de biomasa corporal por efecto de desoves también en primavera (Aizpun de Moreno et al. 1971).

Para el Golfo San Matías, Río Negro (Figura 1), el estudio del ciclo reproductivo ha sido descripto por Calvo et al. (1998), indicando una evacuación gamética en los meses de julio y agosto con emisiones menores hasta octubre. Oehrens Kissner y Kroeck (2005) definieron un período de emisión gamética en enero-febrero a partir de variaciones en el índice de condición y del análisis histológico de mejillones provenientes de un banco del área de El Sótano. En el trabajo analizaron el período noviembre-febrero e infirieron la existencia de un período de desove previo al comienzo de estudio por presencia de alto porcentaje de individuos evacuados. El período de máxima captación de postlarvas registrado en este golfo ocurre a fines de septiembre y principios de octubre (Pascual y Zampatti 1998, RMCP 2013). Por su parte, Elvira et al. (2000) evaluaron la captación en el bajo Olivera y encontraron dos picos de asentamiento, uno en el mes de octubre y otro en abril. Según los autores, el pulso útil para fines productivos lo constituye el del mes de octubre con un rendimiento máximo de 1.010 juveniles $\mathrm{m}^{-1}$. Múltiples experiencias productivas realizadas en Puerto Lobos indican un asentamiento masivo y exitoso en colectores dispuestos en el ambiente natural durante el mes de setiembre (Agulleiro com. pers.) ${ }^{1}$.

En el Golfo San José, Chubut (Figura 1), se realizaron dos estudios sobre el comportamiento reproductivo del mejillón. El primero de ellos se llevó a cabo entre 1976 y 1978 en ambientes infralitorales del llamado banco San Román y El Riacho (Trancart 1978), que permitió observar una estrecha relación del ciclo sexual con la temperatura, y por ende, con las estaciones del año. El ciclo de maduración en los animales adultos comienza a mediados del verano y se extiende hasta el mes de julio-agosto. En este último mes comienza el período de desove que se prolonga hasta diciembre, concentrándose el principal pulso en agosto o septiembre y uno secundario en noviembre o diciembre. El segundo estudio encontró que en mejillones del mesolitoral y el infralitoral del golfo las emisiones de gametas se concentraban básicamente durante los meses de septiembre a diciembre (Bala 1989).

El análisis de la captación sobre colectores en el Golfo San José, Chubut (Figura 1), lo realizaron Ruzzante y Toyos de Guerrero (1984) durante dos períodos de estudio. Encontraron una captación máxima en diciembre y enero para el primer período analizado (1978-1980), y captaciones máximas en noviembre, diciembre y enero para el segundo (1980-1982). En el estudio de Lizarralde (1997) se obtuvo como resultado un pico máximo

\footnotetext{
${ }^{1}$ Ignacio Agulleiro, ex productor de mejillón.
} 
de captación en los meses de septiembre y octubre del orden de 1.800 juveniles $\mathrm{m}^{-1} \mathrm{y}$ otro de menor magnitud en marzo y abril de 300 juveniles $\mathrm{m}^{-1}$, aproximadamente. Desde la perspectiva de aprovechamiento para cultivo, la captación dentro del Golfo San José ocurre tanto en colectores construidos con redes boyadas y ancladas al fondo (Ciocco 1995), como también en estructuras rígidas sobre elevadas (Dellatorre 2006). Experiencias realizadas por la Secretaria de Pesca de Chubut en Riacho San José durante 20082016, indican que el asentamiento en colectores en el intermareal presenta rendimientos elevados que permitieran pensar en estrategias de repoblamiento y de fuente abundante de semilla para la cría y engorde. El momento óptimo de colocación de estas redes intermareales seria a fines del invierno para aprovechar el pulso de desove y captación de setiembre y octubre, cuando se obtienen altos rendimientos del orden de los 8.800 juveniles $\mathrm{m}^{-1}$ (Manriquez com. pers.) $)^{2}$. Experiencias de obtención de semilla para fines comerciales, indican que el momento de colocación de colectores para la obtención se semilla sería en el mes de septiembre y octubre en el Riacho San José (Agulleiro com. pers.) ${ }^{3}$.

En el área costera central del Golfo San Jorge (Figura 1), se infirió el ciclo reproductivo del mejillón a partir de las variaciones mensuales del peso seco de individuos adultos (Isola 2017). Según los resultados obtenidos, existen dos períodos de evacuación gamética: una a fines de primavera y comienzo del verano (diciembre/enero) y otra a comienzos del otoño (mayo). En la misma investigación se registró la captación mensual a partir de la identificación de postlarvas de mejillón sobre colectores artificiales dispuestos en el mar (Isola et al. 2018). La captación se concentró mayoritariamente a finales del verano (marzo) con un promedio 2.900 juveniles $\mathrm{m}^{-1}$, registrándose un pico de escasa magnitud a fina- les de la primavera (diciembre) (Isola 2017).

En la ría Deseado, Santa Cruz (Figura 1), el ciclo sexual del mejillón fue estudiado mediante el análisis histológico y citológico, describiendo las células sexuales y analizando su evolución en ejemplares del mesolitoral inferior-infralitoral superior (Vinuesa 1978). La maduración de las células sexuales se inicia en junio y se extiende hasta diciembre. La emisión de las gametas comienza entre fines de noviembre y enero, pero continúa hasta el mes de mayo, con emisiones menores.

En Bahía San Julián, Santa Cruz (Figura 1), Martín et al. (2015) y Sar et al. (2018) analizaron la disponibilidad de larvas de mejillón en el plancton con muestreos mensuales, encontrando mayor abundancia en febrero. El análisis histológico realizado en dos temporadas reproductivas (2014 y 2015) registró un porcentaje máximo de individuos en estado de liberación de gametas en los meses de diciembre y febrero (Sar et al. 2018). Según Zaixso (2008) las máximas captaciones se encuentran en los meses de verano, principalmente entre diciembre y febrero, con un rendimiento máximo promedio del orden de los 340 juveniles $\mathrm{m}^{-1}$.

En el Canal Beagle (Figura 1) el ciclo sexual del mejillón fue estudiado por Tortorelli (1987). Allí se registran prolongados períodos de desoves en primavera (octubre y noviembre) y otoño (mayo y junio) con cortos períodos de reposo. Los estudios de asentamiento de postlarvas sobre colectores indicaron que se produce entre diciembre y febrero, obteniendo muy buenos resultados en términos de biomasa de captación, de unos 7.959 juveniles $\mathrm{m}^{-1}$ de cuerda colectora (Zampatti, 2002).

En las Islas Malvinas (Figura 1), Gray et al. (1997) analizaron el crecimiento y algunos aspectos parasitológicos de muestras provenientes del mesolitoral inferior mediante técnicas histológi-

\footnotetext{
${ }^{2}$ Rubén Manriquez, Secretaría de Pesca de Chubut, Av. Libertad 279, 9103 - Rawson, Argentina.

${ }^{3}$ Ignacio Agulleiro, ex productor de mejillón.
} 
cas. La conclusión principal fue que la especie comienza su desarrollo reproductivo durante fines de invierno y la primavera, mientras que el desove ocurre principalmente entre enero y febrero. No se ha registrado en las islas ningún estudio de asentamiento de mejillón sobre colectores artificiales.

\section{CONCLUSIONES}

En líneas generales, se puede concluir que existe una tendencia a que la captación de las postlarvas de mejillón en colectores artificiales comience durante el mes siguiente o subsiguiente de producido el desove, ya que el período planctónico de las larvas de mejillón es variable (entre 3 y 5 semanas) (Bayne 1976), sujeto a modificarse en función de las características ambientales, promoviendo o retrasando la metamorfosis hasta un período de más 40 días (Bayne 1965). Los estudios analizados muestran que conforme aumenta la latitud el período de desove de los animales se desplaza desde fines de invierno y comienzos de la primavera (julio-agosto, en Golfo San Matías) hasta avanzado el verano (Bahía San Julián). En la mayoría de los casos se registraron ciclos de desove bimodales. Este tipo de ciclo sexual bimodal con dos picos de desove en primavera y otoño fue descripto para $M$. edulis del hemisferio norte (Lutz et al. 1980; Pulfrich 1996).

En la mayoría de los sitios estudiados se registraron desoves durante el verano. En el caso particular del banco Querandí se observa un solo período de desove en primavera. La población de mejillones estudiada allí se encuentra en bancos circalitorales de entre 30 y $50 \mathrm{~m}$ de profundidad, a diferencia de las poblaciones submareales o intermareales estudiadas en los otros sitios. Esta diferencia de hábitat podría tener un efecto sobre el comportamiento estacional de la temperatura y su dinámica como así también en el régimen de alimentación de los individuos (Bayne 1976; Gosling 2003). Se considera que la temperatura tiene un efecto regulatorio sobre el ciclo sexual de los mejillones (Bayne 1965; Seed 1976; Trancart 1978; Bala 1989; Seed y Suchanek 1992; Gosling 2003). La reproducción está íntimamente ligada con la temperatura del agua, y las variaciones anuales en ella producen invariablemente diferencias en la maduración gamética y el desove. Por otra parte, el rol de la temperatura sobre la duración de la vida larvaria de los mejillones ha sido también ampliamente estudiada (Bayne 1965; Beaumont y Budd 1982; Ruiz et al. 2008; Martel et al. 2014). Por estas razones, cualquier tipo de estudio que analice la reproducción y captación de mejillón en colectores debe considerar la temperatura como una variable ambiental fundamental para ser relevada.

El período de mayor biomasa de asentamiento de las larvas transcurre a comienzos de la primavera en el Golfo San José y los sitios ubicados al norte de él. Hacia el sur, a partir del Golfo San Jorge, las captaciones ocurren durante el verano. Una tendencia que se observa en los golfos patagónicos es que existe un segundo período de captación hacia fines del verano y comienzos del otoño. Este pico de captación suele ser menos abundante que el registrado durante la primavera (Lizarralde 1997; Elvira 2002), a excepción de lo que ocurre en el Golfo San Jorge donde el mayor asentamiento se observa a fines del verano (Isola 2017).

Más allá del rol de los factores ambientales sobre la fisiología reproductiva de los mejillones, la captación en colectores artificiales es un proceso que reviste una elevada complejidad, donde intervienen elementos de distinta naturaleza como el material de fabricación (Cáceres-Martínez et al. 1994), el tiempo de colocación previo al asentamiento (Peteiro et al. 2007), la profundidad (Peteiro 2009; Yildiz y Beber 2010), factores ambientales (Toupoint et al. 2012; Martel et al. 2014), la competencia interespecífica (Gribben et al. 2011), las condiciones hidrodinámicas y de competencia por el espacio (Fuentes-Santos y Labarta 2015), entre otras tantas variables. Por último, la gran variabilidad interanual que presen- 
tan los fenómenos de reclutamiento y asentamiento post-larvario del mejillón debida a las variaciones ambientales descriptas, hacen que este fenómeno sea difícil de estimar con precisión a partir de análisis que solo contemplen períodos anuales. Por este motivo, es conveniente realizar monitoreos durante varios años para detectar las variaciones y así planificar de mejor manera la factibilidad y volumen de aporte de juveniles al cultivo de mejillón. Se requerirán futuros estudios que amplíen el conocimiento existente sobre los aspectos reproductivos y de asentamiento postlarvario que contemplen muestreos simultáneos en las distintas ubicaciones geográficas.

\section{AGRADECIMIENTOS}

Quisiera agradecer a la Lic. M. Eugenia Lopez, Lic. Víctor Fernández; Lic. Mirian Elvira, Dr. Gaspar Soria, Vet. César Gentile, Lic. Cecilia Castaños, Lic. Rubén Manriquez y a la Dra. Cecilia Alvarado, por su ayuda en la búsqueda de bibliografía y aporte documental. A la Lic. Nair Ruiz, Lic. Romina Verga, Lic Javier Tolosano y al Dr. Martín Varisco por su ayuda en la tareas de elaboración cartográfica y edición de imágenes. Finalmente, quisiera agradecer a los revisores anónimos cuyos valorables aportes han contribuido a ampliar y mejorar el artículo.

\section{REFERENCIAS}

Aizpun de Moreno J, Moreno VJ, Malaspina AM. 1971. Estudio sobre el mejillón (Mytilus platenis d`Orb) en explotación comercial del sector bonaerense, Mar Argentino. II. Ciclo anual en los principales componentes bioquímicos. CARPAS. 6 (21): 1-15.

Argentina InNOVAdora. 2020. Ministerio de Ciencia, Tecnología e Innovación Productiva
Secretaría de Planeamiento y Políticas en Ciencia, Tecnología e Innovación Productiva. 2013. Plan Nacional de Ciencia, Tecnología e Innovación: Lineamentos estratégicos 20122015. 99 p.

Astorga MP, Cardenas L, Vargas J. 2015. Phylogenetic approaches to delimit genetic lineages of the Mytilus complex of South America: how many species are there? J. Shellfish Res. 34 (3): 919-930.

BALA JL. 1989. Biología y ecología del mejillón en el gofo San José, provincia de Chubut [Tesis doctoral]. Universidad Nacional de la Plata. 288 p.

BAYNE BL. 1965. Growth and the delay of metamorphosis of the larvae of Mytilus edulis (L.). Ophelia. 2: 1-47.

BAYNE BL. 1976. Marine mussels: their ecology and physiology. En: BAYNE BL, editor. International Biological Programme. Cambridge University Press: 81-115.

BEAumont AR, BudD MD. 1982. Delayed growth of mussel (Mytilus edulis) and scallop (Pecten maximus) veligers at low temperatures. Mar. Biol. 71: 97-100.

Cáceres-Martínez J, Robledo J, Figueras Huerta A. 1994. Settlement and post-larvae behaviour of Mytitus galtoprovincialis: field and laboratory experiments. Mar Ecol Progr Series. 112: 107-117.

Calvo J, Morriconi E, Orler PM. 1998. Estrategias reproductivas de moluscos bivalvos y equinoideos. En: BoschI E. editor. El Mar Argentino y sus recursos pesqueros. Tomo 2. Los moluscos de interés pesquero. Cultivos y estrategias reproductivas de bivalvos y equinoideos. Mar del Plata: Instituto Nacional de Investigación y Desarrollo Pesquero (INIDEP). p. 195-231.

Castellanos DE ZJA. 1957. Los mitílidos argentinos. Buenos Aires. Ministerio de Agricultura y Ganadería, Departamento de Investigaciones Pesqueras. $12 \mathrm{p}$.

ChIPPERFIEld PNJ. 1953. Observations on the 
breeding and settlement of Mytilus edulis (L.) in British waters. J Mar Biol Assoc UK. 32: 449-476.

Ciocco NF. 1995. Marisquería mediante buceo en el Golfo San José y primeras experiencias privadas de cultivo de bivalvos en los Gofos San José y Nuevo. Fundación Patagonia Natural. Plan de manejo integrado de la zona costera patagónica. Informe Técnico $\mathrm{N}^{\mathrm{o}} 2.46 \mathrm{p}$.

DeLl RK. 1964. Antarctic and subantarctic Mollusca: Amphineura, Scaphopoda and Bivalvia. Discovery Reports. 33: 93-250.

Dellatorre FG. 2006. Captación de semilla de mejillón Mytilus edulis platensis (D'Orb 1846) sobre colectores artificiales: Sistema tradicional vs. estructuras rígidas sobreelevadas [resumen]. En: VI Jornadas Nacionales de Ciencias del Mar, Puerto Madryn. p. 167.

Elvira M, Pascual MS, Fernández Cartés V. 2000. Captación masiva de juveniles del mejillón, Mytilus edulis platensis, en el Bajo Oliveira (Golfo San Matías): temporada 199899 y 1999-20 [resumen]. En: IV Jornadas Nacionales de Ciencias del Mar, Puerto Madryn. p. 59.

[FAO] ORganización de las Naciones Unidas para la Alimentación y la Agricultura. 2019. Estadísticas de pesca y acuicultura. Anuario 2017. Roma. 253 p.

FuENTES-SANTOS I, LABARTA U. 2015. Spatial patterns of larval settlement and early post-settlement survivorship of Mytilus galloprovincialis in a Galician Ría (NW Spain). Effect on recruitment success. Regional Studies in Marine Science. 2: 1-10.

GosLing E, editor. 2003. Bivalve Molluscs: Biology, Ecology, and Culture. Fishing News Books. Blackwell Publishing. 443 p.

Gray AP, SEEd R, Richardson CA. 1997. Reproduction and growth of Mytilus edulis chilensis from the Falkland Islands. Scientia Marina. 61 (2): 39-48.

Gribben PE, Jeffs AG, de Nys R, Steinberg PD. 2011. Relative importance of natural cues and substrate morphology for settlement of the New Zealand Greenshell TM mussel, Perna canaliculus. Aquaculture. 319: 240-246.

Isola T. 2017. Aspectos tecnológicos y bio-ecológicos del asentamiento de mejillón, Mytilus edulis platensis, sobre colectores artificiales [Tesis doctoral]. Bahía Blanca: Universidad Nacional del Sur. 153 p.

Isola T, VArisco M, CAzzaniga N. 2018. Implanted byssal hairs on postlarval shells of Mytilus edulis platensis (Bivalvia: Mytilidae) from Patagonia. Zoologia. 35: 1-5.

Kamermans P, CAPelle J. 2019. Provisioning of mussel seed and its efficient use in culture. En: Smaal A, Ferreira JG, Grant J, Petersen JK, STRAND O, editores. Goods and services of marine bivalves. Cham: Springer. p. 27-49.

Lasta ML, Parma AM, Pascual MS, Zampatti EA. 1986. Consideraciones sobre la explotación del mejillón (Mytilus platensis) en la costa bonaerense. Resultados de la campaña de prospección del "Banco Faro Querandí" (junio 1981). Rev Inv Desarr Pesq. 6: 45-55.

LIZARRALDE ZI. 1997. Monthly changes in mussel spat (Mytilus edulis platensis) settlement on artificial collectors in San Jose gulf, Argentina. Biología Pesquera. 26: 43-48.

LUBET P. 1959. Recherches sur le cycle sexuel et l'émission des gamètes chez les Mytilides et les Pectinidés (Mollusques bivalves). Revue des Travaux de 1'Institut des Pêches Maritimes. 23: 389-548.

Lutz RA, Incze LS, Porter B, Stotz JK. 1980. Seasonal variation in the condition of Raftcultivated mussel (Mytilus edulis L.). Proc World Maricul Soc. 11: 262-268.

Martel AL, Tremblay R, Frédéric Olivier NT, MYrAND B. 2014. Veliger size at metamorphosis and temporal variability in prodissoconch II morphometry in the blue mussel (Mytilus edulis): Potential impact on recruitment. J Shellfish Res. 33 (2): 443-455.

Martín JP, Sar A, Caminos C, Fernández R, Kroeck M, Morsán E. 2015. Ciclo reproduc- 
tivo y reclutamiento del mejillón Mytilus edulis platensis en la Bahía de San Julián (Patagonia Austral Argentina). IX Jornadas Nacionales de Ciencias del Mar, Ushuaia. Resúmenes: p. 379.

Oehrens Kissner EM, Kroeck MA. 2005. Estimación de la talla de primera madurez sexual del mejillón, Mytilus edulis platensis (Mollusca: Bivalvia) en el Golfo San Matías. IBMP Serie Publicaciones, 2005, IV: 21-44.

Panné Huidobro S. 2016. Producción por acuicultura en Argentina 2016. Dirección de Acuicultura-DNPyP, Subsecretaría de Pesca y Acuicultura, Ministerio de Agroindustria, Argentina. 6 p. <http://www.fao.org/fishery/ countrysector/naso_ argentina/es\#tcN700C5> [Actualizado junio 2018; citado noviembre 2019].

Pascual MS, Zampatti E. 1998. El cultivo de moluscos bivalvos. En: Boschi E, editor. El Mar Argentino y sus recursos pesqueros. Tomo 2. Los moluscos de interés pesquero. Cultivos y estrategias reproductivas de bivalvos y equinoideos. Mar del Plata: Instituto Nacional de Investigación y Desarrollo Pesquero (INIDEP). p. 167-193.

Penchaszadeh PE. 1971. Estudios sobre el mejillón (Mytilus platensis d'Orbigny) en explotación comercial del sector bonaerense, Mar Argentino. I- Reproducción, crecimiento y estructura de la población. Documento Técnico 12. CARPAS. 15 p.

Penchaszadeh PE. 1974. Ecología del mejillón Mytilus platensis d'Orbigny de bancos circalitorales [Tesis doctoral]. Universidad de Buenos Aires. 188 p.

Peteiro L. 2009. Patrones de asentamiento y reclutamiento de Mytilus galloprovincialis en la Ría de Ares-Betanzos e implicaciones para su cultivo en batea [Tesis doctoral]. Universidad de la Coruña. 152 p.

Peteiro L, Filgueira R, Labarta L, FernándezREIRIZ MJ. 2007. Effect of submerged time of collector ropes on the settlement capacity of
Mytilus galloprovincialis L. Aquaculture Res. 38: 1679-1681.

Pulfrich A. 1996. Attachment and settlement of postlarval mussels (Mytilus edulis L.) in the Schleswlgholstein Wadden Sea J Sea Res. 36: 239-250.

[RMCP] Red de Fortalecimiento para la MAricultura Costera Patagónica. 2013. Relevamiento de la actividad de la maricultura en la Patagonia Argentina. Informe Técnico I. Puerto Madryn. 222 p.

Ruiz M, TArifeño E, Llanos-Rivera A, Padget C, CAmpos B. 2008. Temperature effect in the embryonic and larval development of the mussel, Mytilus galloprovincialis (Lamarck, 1819). Rev Biol Mar Oceanog. 43 (1): 51-61. Ruzzante DE, Toyos DE Guerrero MA. 1984. Captación de mitílidos sobre colectores. I. Variaciones en la captación de Mytilus platensis D'Orb. y de Aulacomya ater (Molina). Physys A. 42 (102): 55- 62.

Sar A, Martin J, Fernández R, Caminos C. 2018. Ciclo reproductivo y reclutamiento del mejillón Mytilus edulis platensis en Bahía San Julián (Santa Cruz, Argentina). Informe Científico Técnico UNPA, 2: 33-51.

SEED R. 1976. Ecology. En: BAYNe BL, editor. Marine mussels: their ecology and physiology. Cambridge: Cambridge University Press. p. 13-65.

SEED R. 1990. Taxonomic and evolutionary relationships within the genus Mytilus. En: MorTON B, editor. The Bivalvia. Proceedings of the Memorial Symposium in Honour of Sir Charles Maurice Yonge, Edinburgh, 1986. Hong Kong: Hong Kong University Press (Asian Marine Biology 7). p. 97-110.

Seed R, SuchaneK TH. 1992. Population and community ecology of Mytilus. En: GosLING EM, editor. The mussel Mytilus: ecology, physiology, genetics and culture. Amsterdam: Elsevier Science Publishers BV. p. 87-169.

Tortorelli MC. 1987. Contribución al estudio de los ciclos reproductivos del mejillón pata- 
gónico, Mytilus chilensis Hupé, y de la cholga, Aulacomya ater (Molina), en el canal de Beagle [Tesis doctoral]. Universidad de Buenos Aires. 257 p.

TrAnCART M. 1978. Biologie et possibilités d'exploitation de Mytilus platensis (D'Orb.) dans le Golfe San Jose, Péninsule Valdes, Argentine. Thèse de Doctorat de 3ème Cycle, Océanologie. U.E.R. des Sciences de la Mer et de L'Environnement, Université d'Aix-Marseille-II. 86 p.

Toupoint NL, SOlOMON-Gilmore F, Bourque B, Myrand F, Pernet F, Olivier F, Tremblay R. 2012. Match/mismatch between the Mytilus edulis larval supply and seston quality: effect on recruitment. Ecology. 93: 1922-1934.

VinUESA JH. 1978. Ciclo gonadal y primera madurez sexual del mejillón patagónico Mytilus edulis chilensis Hupé, en Puerto Deseado. Physis A. 38 (95): 35-47.

WiJsman JWM, Troost K, FAng J, Roncarati A. 2019. Global Production of Marine Bivalves. Trends and Challenges. En: SMAAL A, FERREIRa J, Grant J, Petersen J, Strand Ø, editores. Goods and Services of Marine Bivalves.
Cham: Springer. p. 7-27.

Yildiz H, BERBER S. 2010. Depth and seasonal effects on the settlement density of Mytilus galloprovincialis L. 1819 in de Dardanelles. J Animal Vet Advances. 4: 756-759.

ZAIXso HE. 2008. Producción de semilla de mejillón. Informe Técnico a pedido de la Agencia de Desarrollo Sustentable de Puerto San Julián. Puerto San Julián, Santa Cruz, Argentina. $103 \mathrm{p}$.

ZAMPATTI E. 2002. Apoyo para la implementación desarrollo y fomento del cultivo de mejillón en la provincia de Tierra del Fuego. Informe Final. CFI. 117 p.

Zbawicka A, Trucco MI, Wenne R. 2018. Single nucleotide polymorphisms in native South America Atlantic coast populations of smooth shelled mussels: hybridization with invasive European Mytilus galloprovincialis. Genet Sel Evol. 50: 5. doi: 10.1186/s12711-018-0376-Z

Recibido: 27-08-2019

Aceptado: 25-11-2019 